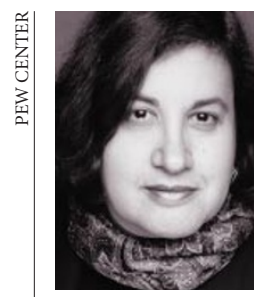

to cut carbon emissions "We're making a lot of progress here," says Dan Lashof, head of the climate-change programme at the Natural Resources Defense Council, pointing to Claussen: targets recent bipartisan calls in for emissions the Congress for reducmust be realistic. tions in power-plant emissions.

Jennifer Morgan, director of the World Wildlife Fund's climate-change campaign, also believes the Kyoto Protocol can work. "We should be focusing on a domestic plan to meet the targets, not on changing them," she says.

But the Global Climate Coalition, the industry group that has opposed binding action to cut carbon emissions, said that Claussen's statements bore out its early warnings about the Kyoto Protocol. "We agree with her that the targets are simply unrealistic," says Connie Holmes, chair of the coalition's board. "In the last three years, they have become more unrealistic - and not just for the United States.”

i http://www.pewclimate.org/media/

transcript_riia.html

\title{
Proposal for US patent office could help cut waiting time
}

Paul Smaglik, Washington

Anyone who has recently applied for a US patent - particularly in high-tech fields or biotechnology - will have waited a long time to learn of their request's fate. A congressman is now proposing to address this problem by allowing the United States Patent and Trademark Office (USPTO) to keep all the fees that it raises.

The move would help to increase the number of examiners - without this, warns the office, the backlog of applications is likely to grow even longer. Applications have increased by $12 \%$ in the past year alone, and the time for processing each one has grown from 18 months in 1995 to 26 months now.

Up to now, the patent office has always handed over a portion of its income to the government. But the precise percentage has varied, creating difficulties with long-term hiring commitments.

In the next financial year, which begins in October, the USPTO expects to take in about $\$ 1.2$ billion. But the House, Senate and President Bill Clinton's administration disagree over how much the agency should keep. The House is suggesting $\$ 905$ million, whereas the Senate last week voted to allow it to retain all but $\$ 33$ million; the president's budget calls for the USPTO to give up \$113 million.

Representative Howard Coble (Republican, North Carolina) has put forward a bill that would allow the USPTO to spend everything it earns. The administration has not commented on this proposal. But in a letter to Coble earlier this summer, USPTO commissioner Todd Dickinson warned of dire consequences if the House's budget were adopted, as the office would have to impose a hiring freeze and reduce overtime.

Demand for patents is especially heavy in genomics, where there is already a backlog of 20,000 applications for patents on genes, says Lila Feisee, a spokesperson for the Biotechnology Industry Organization.

Analysing these applications requires more people and better software, adds Feisee, a former USPTO examiner. "There's such an outcry over gene patents now that it's important to do it right," she says.

\section{Physicists celebrate detection of elusive 'final' particle}

\section{Colin Macilwain, Washington}

High-energy physicists are declaring 'full house' after detecting the last remaining basic building block of matter predicted by the Standard Model. A team at Fermilab outside Chicago has seen the elusive tau neutrino for the first time.

The DONUT (Direct Observation of the $\mathrm{Nu}$ Tau) team of 54 physicists from the United States, Japan, South Korea and Greece announced last week that they had found the kinked, millimetre-long decay tracks of four tau leptons - produced when an iron nucleus and a tau neutrino collide - after analysing millions of such possible events.

The Standard Model of fundamental particles and their interactions predicts the existence of 12 'fermions' - the basic building blocks of matter - and four 'vector bosons', which carry force. The tau neutrino, which belongs to a class of fermions called leptons, was the last to evade detection.

"Finding the tau neutrino is very important and exciting," says Martin Perl of Stanford University, California, who won the 1995 Nobel Prize in Physics for his discovery of the tau lepton. "DONUT was not an easy experiment, and now it opens a whole new world. We might have a chance of learning more about all other particles."

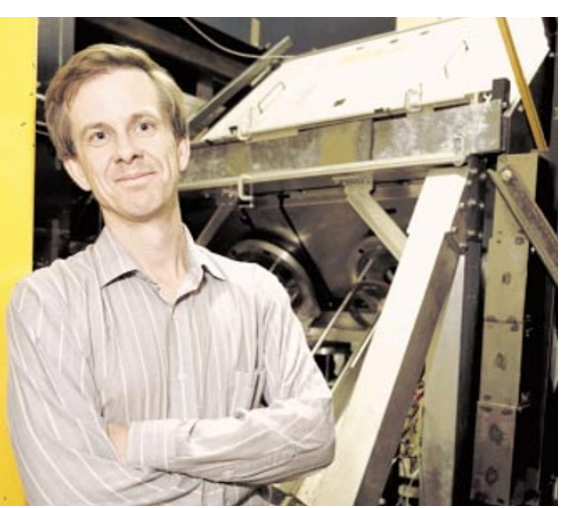

DONUT delight: spokesman Lundberg describes discovery as 'the proverbial needle in a haystack'.

In particular, the finding may assist the burgeoning field of neutrino physics. Early results from the SuperKamiokande experiment in Japan suggest that neutrinos may have mass (see Nature 391, 123; 1998). To test this, physicists in Japan, Europe and the United States are planning experiments to observe the oscillation of one type of neutrino to another.

The DONUT experiment began in 1997, by sending a high-energy proton beam from Fermilab's main particle accelerator, the Tevatron, into a block of tungsten.
Researchers filtered a beam of neutrinos out of the resulting mêlée, and this was passed through a target of iron sandwiched with layers of emulsion.

The DONUT team identified 1,000 events of interest and took the emulsion plates, which work like three-dimensional photographic film, to Nagoya University in Japan for analysis. "It was the proverbial needle in a haystack," says Fermilab's Byron Lundberg, spokesman for the experiment.

High-energy physicists are now switching their attention to the Higgs mechanism, an extension to the Standard Model, which explains how particles acquire mass. This involves exchanges of a particle called the Higgs boson, the search for which will resume at a revamped Tevatron next year. CERN, the European Laboratory for Particle Physics near Geneva, will join the hunt in 2005 with its Large Hadron Collider.

Meanwhile, some researchers are excited by 'supersymmetry', a theory outside the Standard Model. This predicts that every fermion has a supersymmetric boson counterpart, and vice versa. Experiments at CERN's Large Electron-Positron collider have hinted at a counterpart to the bottom quark - although previous hints have turned out to be statistical fluctuations. 